\title{
Justice au travail - Lutte à armes égales
}

Jo Carby-Hall

\section{(2) OpenEdition}

Journals

Édition électronique

URL : https://journals.openedition.org/rdctss/1695

DOI : $10.4000 /$ rdctss. 1695

ISSN : 2262-9815

Éditeur

Centre de droit comparé du travail et de la sécurité sociale

Édition imprimée

Date de publication : 1 avril 2019

Pagination : 234-237

ISSN : 2117-4350

\section{Référence électronique}

Jo Carby-Hall, « Justice au travail - Lutte à armes égales », Revue de droit comparé du travail et de la sécurité sociale [En ligne], 1 | 2019, mis en ligne le 01 novembre 2021, consulté le 13 novembre 2021. URL : http://journals.openedition.org/rdctss/1695; DOI : https://doi.org/10.4000/rdctss.1695

\section{(c) $(1)(9)$}

Revue de droit comparé du travail et de la sécurité sociale est mise à disposition selon les termes de la Licence Creative Commons Attribution - Pas d'Utilisation Commerciale - Pas de Modification 4.0 International. 


\section{JO CARBY-HALL}

UNIVERSITÉ DE HULL

\section{JUSTICE AU TRAVAIL - LUTTE À ARMES ÉGALES}

Le gouvernement de Teresa May a l'intention de renforcer les droits des personnes vulnérables occupées dans des emplois atypiques pour démontrer sa volonté de s'intéresser aux inégalités. Lorsque Teresa May a été nommée Premier Ministre, son intention était de gérer un pays ${ }^{1}$ " où tous les individus sont soumis à la même règle du jeu et où chaque personne, quel que soit son milieu d'origine, puisse réaliser ses ambitions ». Au titre de la nouvelle législation prévue par le gouvernement, toute personne employée par un contrat «zero-hour » ou dans la gig-économie ou un travailleur mis à disposition par une agence de recrutement se verront octroyer une protection en vertu d'une série de réformes sur ces modes d'emploi. Les travailleurs susmentionnés jouiront de certains droits auxquels ils n'avaient pas accès jusqu'alors.

Ces réformes se basent sur les conclusions et sur certaines des 53 recommandations de la «Taylor Review $»^{2}$, parmi lesquelles on trouve une stratégie nationale ayant pour but d'assurer un bon emploi pour tous et «dont le gouvernement doit assumer la responsabilité ». L'expression «bon emploi» signifie, entre autres, la qualité de l'emploi, les conditions de travail, à savoir l'équilibre travail-vie privée et un salaire adéquat. Le gouvernement devrait assurer à chaque travailleur une protection de base et la garantie de possibilités de progression dans l'emploi.

Le 17 décembre 2018, le gouvernement a publié son «Good Work Plan » en réponse aux recommandations de la Taylor Review. Le Right Honourable George Clark, Secretary of State for Business, Energy and Industrial Strategy a estimé que ce document ${ }^{4}$ était la " plus considérable des améliorations des droits des travailleurs depuis plus d'une génération », qu'il «constituait un élément clé pour établir un marché du travail récompensant le bon travail, reconnaissant les bons employeurs et stimulant le potentiel de productivité et des salaires au Royaume-Uni ».

Nous allons ici procéder à une analyse des modifications que le "Good Work Plan» apportera aux droits des travailleurs. Notons d'emblée que le gouvernement n'a posé qu'un nombre très limité de règles pour rendre effectives les réformes mentionnées dans le «Good Work Plan ». A ce jour, pour la majorité des réformes proposées, le projet de loi et le calendrier n'ont pas encore été soumis à la consultation.

1. Les entreprises seront soumises à l'obligation de fournir à leurs travailleurs une déclaration stipulant leurs droits et avantages dès le premier jour de leur entrée en fonction

1 Source BBC News - Politics 5 octobre 2018.

2 Pour une analyse de la 'Taylor Review' voir Jo Carby-Hall, RDTSS, n² 2018-1.

3 Policy Paper "Good Work Plan" 14 décembre 2018. Com. 9757.

https://www.gov.uk/governmen/pubications/good-work-plan

4 Source: https://www.bbc.co.uk/news/business-46586623 
avec en plus la mention de leurs droits aux congés payés, congés maladie, maternité et paternité et au partage du congé parental ${ }^{5}$.

2. Il est aussi proposé d'établir un droit qui mette tous les travailleurs sur un pied d'égalité en comblant un vide juridique connu sous le terme de "Swedish Derogation Rule », qui donne aux entreprises le droit de rémunérer les travailleurs recrutés en agence moins que leurs salariés permanents. Au titre des «Agency Workers Regulations » de 2010, les travailleurs recrutés en agence, employés pour une durée de 12 semaines ou plus par le même employeur ont droit aux mêmes salaires et avantages que le personnel permanent grâce à l'inclusion de la «Swedish Derogation » dans la Directive européenne à la demande du gouvernement suédois, des exemptions sont permises. Les travailleurs temporaires recrutés en agence peuvent donc être employés directement par l'agence plutôt que par l'entreprise utilisatrice. Cela leur permet d'être rémunérés entre deux affectations et ainsi de ne pas toucher le même salaire ni d'avoir les mêmes avantages que le personnel permanent. Bien que la pratique soit légale, les critiques avancent l'argument que ce vide juridique instaure deux niveaux dans le personnel et ouvre la porte à l'exploitation des travailleurs d'agence. La Présidente de la «Law Society » d'Angleterre a émis l'opinion que « les travailleurs peuvent maintenant être confortés à l'idée qu'ils ont le droit d'être traités et rémunérés sur un pied d'égalité avec leurs collègues employés directement par leur employeur et que ce droit est légalement exécutoire ${ }^{6}$.

3. Le gouvernement suggère de promulguer une législation qui offre protection aux travailleurs d'agence dans les cas où le paiement de leur salaire est suspendu ou si des déductions injustifiées sont retenues par des "entreprises parapluies » qui très souvent dans ce secteur paient le salaire des travailleurs d'agence.

4. Les travailleurs auront droit à une information détaillée sur le type de contrat par lequel ils sont employés, sur les modalités de paiement de leur salaire et sur le montant minimum à percevoir. Là où c'est un intermédiaire qui doit payer le salaire, le travailleur doit être informé du montant des frais, des déductions à la source imposées par l'intermédiaire ainsi que de toute déductions pour frais de trajet, de logement, etc. De plus, la législation obligera l'entreprise à calculer une estimation du salaire final toute déduction faite.

5. La législation prévoit également une augmentation du montant maximal de l'amende imposée aux employeurs par l'Employment Tribunal; amende qui passe de 5000 livres sterling à 20000 livres, si le Tribunal juge que l'employeur a fait preuve envers ses salariés de "malveillance, méchanceté, négligence aggravée (violations graves). Le processus comprendra aussi une peine connue sous les termes de «naming and shaming » c'est-à-dire que les manœuvres de l'employeur feront l'objet d'une publicité. Au cas où un employeur traduit devant un tribunal refuserait de payer une compensation au travailleur lésé qui a gagné le procès, celui-ci pourra présenter une requête auprès du Business, Energy and Industrial Strategy Department qui rendra public son comportement (" he will be fined and named publicly»). Le travailleur devra remplir un formulaire fourni par l'EmploymentTribunal Penalty Enforcement. Dans ce cas, l'employeur recevra une

5 The Employment Rights (Employment Particulars and Annual Leave) (Amended) Regulations 2018, $\mathrm{n}^{\circ} 1378$ qui apporte un amendement aux sections 1 à 7B de l'Employment Rights Act, 1996 et entrera en vigueur le 6 avril 2020.

6 II est prévu que la législation sur la «Swedish Derogation » soit débattue au Parlement début de 2019 et qu'elle entre en vigueur le 6 avril 2020. 
notification le sommant de payer le travailleur sous 28 jours. S'il s'abstient d'obtempérer, on lui infligera une amende et il est possible « qu'il soit cité par le gouvernement ${ }^{7}$ ».

6. Le gouvernement accordera un autre droit aux travailleurs: les entreprises devront calculer le paiement des congés annuels sur la base de cinquante-deux semaines ${ }^{8}$ au lieu de douze semaines actuellement. Pour les travailleurs saisonniers et les travailleurs atypiques dont la paie varie parce qu'ils n'ont pas d'horaires de travail normaux, ou, même s'ils en ont, leur temps de travail, les jours ou les heures varient, cela leur permettra de percevoir le paiement des congés qui leur est dû . Les travailleurs à temps partiel ont droit à autant de congés que les travailleurs à temps complet, calculés au pro rata. Pour ceux qui travaillent par poste, il est généralement plus facile de faire le calcul sur la base des postes non ouvrés. Quant aux travailleurs intermittents ou occasionnels ou ceux qui ont des horaires irréguliers, il est plus facile de calculer leurs droits aux congés payés proportionnellement aux heures ouvrées. Tout travailleur a droit à 5,6 semaines de congés annuels, ce qui équivaut à $12,07 \%$ des heures ouvrées annuellement. Le chiffre de 12,7\% s'obtient en divisant 5,6 semaines de congés annuels par 46,4 semaines (c'est-à-dire 52 semaines moins 5,6 semaines). Les 5,6 semaines sont exclues du calcul car elles ne sont pas ouvrables, elles n'accumulent donc pas de congés ${ }^{10}$.

7. Le législateur se propose aussi d'accorder aux travailleurs employés sous contrat à horaires flexibles, le droit de poser une demande de contrat plus stable à horaires fixes qui leur assure la sécurité financière dès lors qu'ils ont travaillé dans un emploi atypique ou vulnérable pendant 26 semaines. II est cependant admis que les entreprises ne soient pas obligées d'accéder automatiquement à cette demande.

8. Le gouvernement s'est engagé à légiférer pour apporter des clarifications au statut de l'emploi d'une personne - employé, ouvrier ou travailleur indépendant - qui refléteront la réalité actuelle des relations de travail. Tenant compte de la complexité de ce sujet due aux nombreux tests créés par les tribunaux au cours des siècles derniers ${ }^{11}$, le gouvernement et le législateur auront la tâche difficile et peu enviable de clarifier le statut de l'emploi. Cette clarification aiderait les employeurs à éviter une classification erronée de leur personnel.

9. En lien avec le point précédent on trouve la proposition d'aligner le statut de l'emploi sur la formule utilisée par les services des impôts, c'est-à-dire Her Majesty's Revenue and Customs (HMRC). HMRC définit actuellement le statut de l'emploi d'une manière adaptée à ses besoins. La différence dans l'interprétation engendre la confusion. Si des différences doivent exister, il faudra les réduire autant que possible.

7 Les notifications de peines s'appliquent aux jugements prononcés le 6 avril 2019 ou après. La citation publique s'applique aux jugements enregistrés le 18 décembre 2018 ou après.

8 Regulation 16 des Working Time Regulation, 1998 qui applique la Council Directive 2304/104EC (OJ), nL107 (Amended).

9 Employment Rights (Employment Regulations and Annual Leave (Amendment) regulations 20118, n 1378 qui entrera en vigueur le 6 avril 2020.

10 Source: Advisory, Conciliation and Arbitration Service (ACAS)'s calculation.

11 Pour une analyse détaillée de ces tests voir J. Carby-Hall « New Frontiers of Labour Law :-Dependent and Autonomous Workers» in B. Veneziani et U. Carabellidir (dir.) Du travail salarié au travail indépendant: Permanences et Mutations, Programme SOCRATES, éd. Cacucci,2003, p. 163. 


\section{ROYAUME-UNI}

10. D'autres points doivent faire l'objet d'une législation:

- interdire aux employeurs de procéder à des déductions du salaire de leur personnel du fait qu'ils touchent un pourboire de leurs clients.

- allonger d'une à quatre semaines la période requise pour définir une rupture de la continuité d'emploi pour permettre aux travailleurs d'accumuler des droits quand il y a un écart entre deux affectations.

- repousser le seuil actuel de $19 \%$ à $2 \%$ du nombre de travailleurs requis pour l'organisation d'une procédure d'information et de consultation ou d'un «Work Council»

- obliger les Employment Tribunals à prendre en compte les sanctions imposées aux employeurs déjà sanctionnés par un Employment Tribunal pour des faits identiques, similaires ou comparables.

- introduire des mesures supplémentaires pour éviter que des stagiaires non rémunérés ne fassent le travail d'un membre du personnel.

- réviser les termes de la licence du GLAA pour qu'ils reflètent exactement les droits actuels des travailleurs et les obligations des employeurs.

- donner au Secretary of State for Business, Energy and Industrial Strategy. une responsabilité supplémentaire celle d'assurer la qualité du travail.

Le mécanisme qui assure les droits des travailleurs, la protection des travailleurs vulnérables et des bas salaires contre l'exploitation et qui assure l'amélioration de la qualité du travail existe déjà. II s'agit des inspecteurs de l'Employment Agency Standards (EAS) de I'Equipe pour le Minimum Wage de HMRC, et du Gangmasters and Labour Abuse as Authority (GLAA). Le gouvernement a répondu à la Stratéegie UK Labour Market Enforcement pour 2018 - 2019, définie par le Directeur du Labour Market Enforcement dans un plan détaillé qui vise à s'attaquer à l'exploitation des travailleurs en bas de l'échelle des salaires. Ce plan suggère, entre autres, d'avancer à début de 2019 la création d'un organisme de mise en place des lois pour la protection des travailleurs vulnérables.

Le Good Work Plan qui constitue la partie-clé de la stratégie moderne du gouvernement pour l'industrie est la dernière réponse du gouvernement aux recommandations du Rapport Taylor de 2017 qui a démontré que la force du marché du travail britannique réside dans sa flexibilité mais qu'il fallait prendre en compte tout particulièrement autant la qualité du travail que le nombre d'emplois. Les réformes auront pour effet d'amener les employeurs à raffiner leurs pratiques d'emploi afin de protéger les droits des travailleurs et pour citer le gouvernement "s'assurer que les travailleurs aient accès à un travail juste et convenable, qu'employeurs et travailleurs soient clairement informés pour qu'ils comprennent les relations dans l'emploi et s'assurer que l'application du système soit juste et adaptée à l'objectif.». 